\title{
Animalidad, comunidad e imaginarios de lo trans en la obra visual de Nicola Costantino
}

\author{
Cynthia Francica' (iD) 0000-0001-7440-8090
}

'Universidad Adolfo Ibáñez, Santiago, Chile.admisionstgo@uai.cl

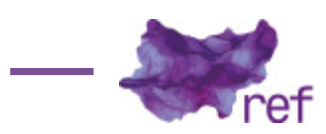
Resumen: En el contexto actual de cuestionamiento radical de la categoría de lo 'humano', un sector
de la producción visual contemporánea investiga los modos en que las subjetividades de género
toman forma no sólo en relación con entornos culturales humanos, sino también a geografías materiales,
naturales e inanimadas. Enfocándome en el caso de la artista argentina Nicola Costantino (Rosario,
1964), propongo que mediante la recuperación de superficies corporales sensuales, táctiles y
afectivamente cargadas, su obra Peletería humana (1996-2003) negocia articulaciones hegemónicas
de la sexualidad y el género e historias comunales de violencia, al tiempo que revisita imaginarios
simbólicos nacionales para explorar y delimitar nuevas corporalidades y subjetividades potenciales
en torno tanto a lo femenino como a lo queer y lo viviente.

Palabras clave: Animalidad; afectos; trans; arte argentino; Nicola Costantino.

\section{Animality, Affect and Trans Imaginaries in the Visual Art of Nicola Costantino}

Abstract: In today's context of radical questioning of the category of the 'human', a sector of contemporary visual arts investigates the ways in which gendered subjects take shape not only in relation to human cultural environments but also to material, natural and inanimate geographies. Focusing on the case of Argentine artist Nicola Costantino (Rosario, 1964), I propose that through the recovery of sensual, tactile and affectively charged bodily surfaces, her artwork Peletería humana (1996-2003) negotiates hegemonic articulations of gender and sexuality and communal histories of violence while revisiting national symbolic imaginaries in order to explore new potential subjectivities and bodily configurations regarding the feminine, the queer and the living.

Keywords: Animality; Affect; Trans; Argentine art; Nicola Constantino.

Animalidade, comunidade e imaginários trans na obra visual de Nicola Costantino Resumo: No atual contexto de questionamento radical da categoria de 'humano', um setor de produção visual contemporânea investiga as formas pelas quais as subjetividades de gênero tomam forma não apenas em relação aos ambientes culturais humanos, mas também às geografias materiais, naturais e inanimadas. Focalizando o caso da artista argentina Nicola Costantino (Rosario, 1964), proponho que, ao recuperar superfícies corporais sensuais, táteis e emocionalmente carregadas, seu trabalho Peletería Humana (1996-2003) negocia articulações hegemônicas de sexualidade e gênero e histórias comunitárias de violência, enquanto revisa o imaginário simbólico nacional para explorar e delimitar novas corporalidades e subjetividades potenciais em torno do feminino, do queer e dos vivos. Palavras-chave: Animalidade; afetos; trans; arte argentina; Nicola Costantino.

En el contexto actual de cuestionamiento radical de la categoría de lo 'humano' y sus límites semióticos, éticos y ontológicos en el ámbito de las Humanidades, la indagación en lo viviente emerge con fuerza inusitada en una serie de obras artísticas latinoamericanas contemporáneas. En línea con el pensamiento de la teórica feminista Rosi Braidotti y su conceptualización de lo humano como "una convención normativa, no intrínsecamente negativa, pero con un elevado poder reglamentario y, por ende, instrumental a las prácticas de exclusión y discriminación" (Rosi BRAIDOTI, 2015, p. 39), un sector de la producción visual contemporánea parece investigar específicamente los modos en que las subjetividades de género toman forma no sólo en relación a entornos cultural 
humanos sino también a geografías materiales, naturales e inanimadas. Así, la urgencia de trazar un territorio de indagación más allá de lo humano para pensar problemáticas de género y sexualidad cobra sentido en el contexto de una renovada atención a los modos en que "el estándar humano [...] funciona transponiendo un particular modo de ser humano en un modelo generalizado, que es categórica y cualitativamente distinto de los otros sexualizados, racializados y naturalizados" (BRAIDOTTI, 2015, p. 39).

Con objeto de abordar los modos en que el arte actual explora esas fronteras, me enfocaré aquí en el caso de la artista argentina Nicola Costantino (Rosario, 1964), quien despliega una particular estética en torno al cuerpo femenino, los imaginarios de lo trans, la animalidad y la abyección. Para este análisis me interesa centrarme en particular en su obra Peletería Humana (1996-2003, Fig. 1), la cual explora la estética y la política del animal para repensar el cuerpo contemporáneo a partir de técnicas de costura y manipulación de peletería. ${ }^{1}$

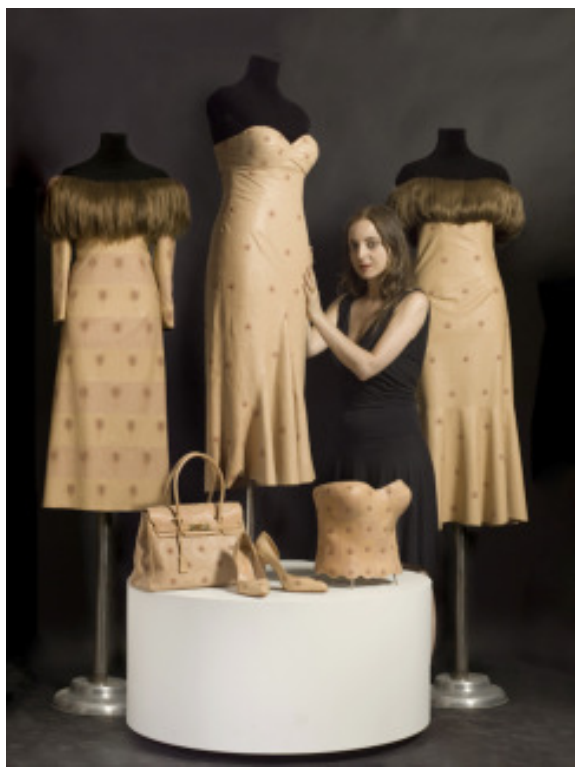

Figura 1. Peletería Humana (Human Furriery), Nicola Costantino, 1996-2003.

\#PraTodoMundoVer Exposición Peletería Humana montada en maniquíes. La foto presenta 3 vestidos de cóctel de hombros descubiertos, de corte recto, color beige arena, con pequeños calcos (como lunares espaciados). El de la izquierda tiene calcos de anos, mangas largas, rayas anchas horizontales alternando tonos oscuros y claros de color beige, y estola de cabello humano color avellana alrededor del escote; el del medio no tiene mangas y tiene calcos de tetillas; $y$ el tercer vestido no tiene mangas, tiene estola, y calcos de tetillas; en frente y abajo, sobre un taburete blanco, se exponen una cartera, un par de zapatos y un corset. Todas las prendas son hechas de la misma tela, con calcos. La artista posa junto a su obra, atrás del taburete, apoyando sus manos en el vestido del medio y mirando hacia la cámara. Ella es blanca, tiene cabello marrón lacio largo, usa lápiz de labios color rojo, y lleva un vestido negro sin mangas con escote.

Peletería Humana consiste en una colección de vestimentas realizadas en base a calcos de partes corporales humanas que, subvirtiendo y desnaturalizando el código estético de la peletería, generó reacciones viscerales al literalizar y explorar los vínculos entre la moda, la muerte y la abyección. Algunas de las vestimentas de moda que componen la pieza incorporan cabello humano real, y consisten en vestidos, corsés, tapados y accesorios como zapatos y carteras (Figs. 2, 3, 4, 5, 6, 7 e 8).

Figura 2. Peletería Humana (Human Furriery), Nicola Costantino, Deitch Projects, Nueva York, 2000.

\#PraTodoMundoVer Exposición Peletería Humana en Galería Deitch Projects, Nueva York. Fotografía tomada desde la calle. Vestidos, sacos, zapatos y carteras expuestos en maniquíes y visibles a través de la vidriera exterior de la galería.

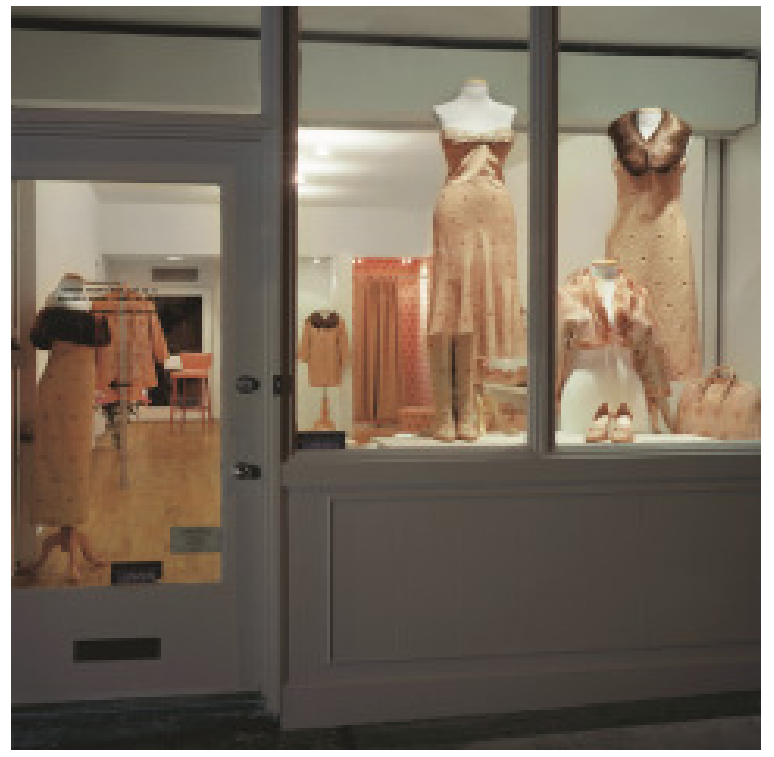

${ }^{1}$ Esta obra puede visionarse en el siguiente link: https://www.nicolacostantino.com.ar/peleteria.php. Los otros trabajos de Costantino comentados pueden accederse también en el sitio web de la artista: https://www.nicolacostantino.com.ar/ home.php 


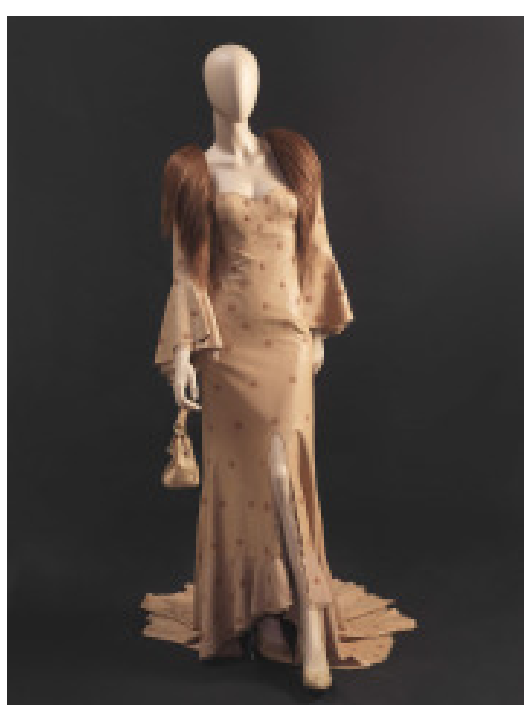

Figura 3. Peletería Humana, Nicola Costantino, 1996-2003.

\#PraTodoMundoVer Vestido largo en silicona cubierto con calcos de tetillas expuesto en maniquí, con tajo en la pierna, mangas largas y pelo natural en los hombros, acompañado de una cartera que cuelga de la mano derecha del maniquí.

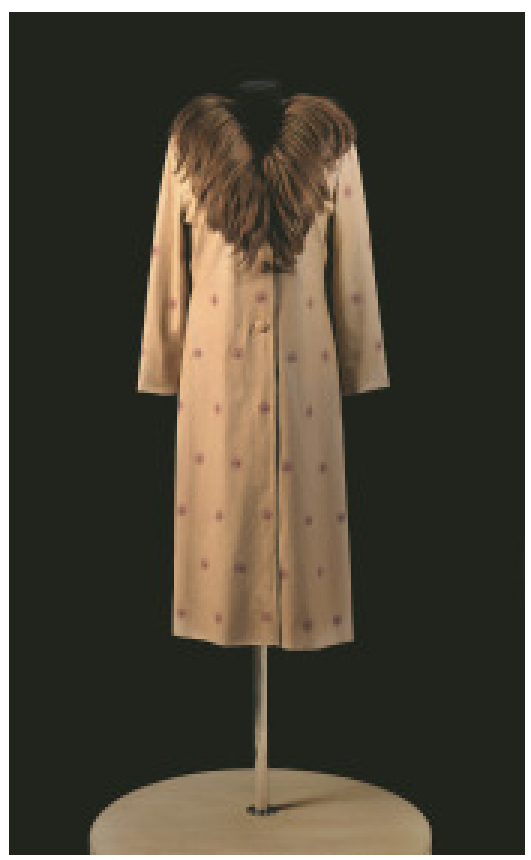

Figura 5. Peletería Humana, Nicola Costantino, 1996-2003.

\#PraTodoMundoVer Saco largo en silicona cubierto con calcos de tetillas montado, con mangas largas y pelo natural en el escote.

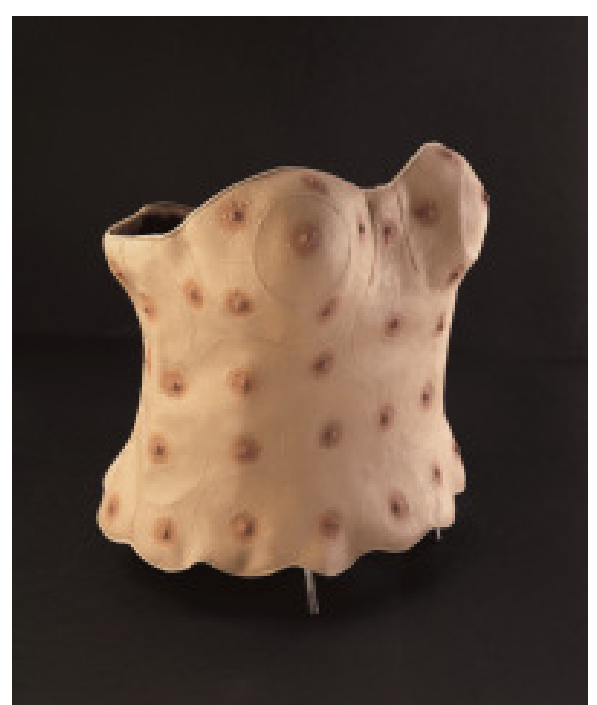

Figura 4. Peletería Humana, Nicola Costantino, 1996-2003.

\#PraTodoMundoVer Corset en silicona con calcos de tetillas montado, cuyos moldes circulares resultan visibles.

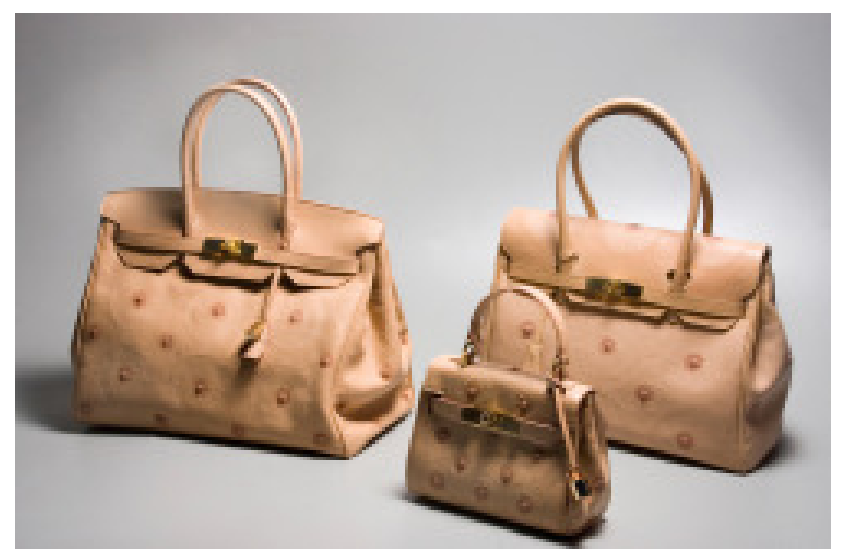

Figura 6. Peletería Humana, Nicola Costantino, 19962003.

\#PraTodoMundoVer Tres carteras de mujer en silicona de diferentes tamaños y diseños similares, cubiertas con calcos de tetillas.

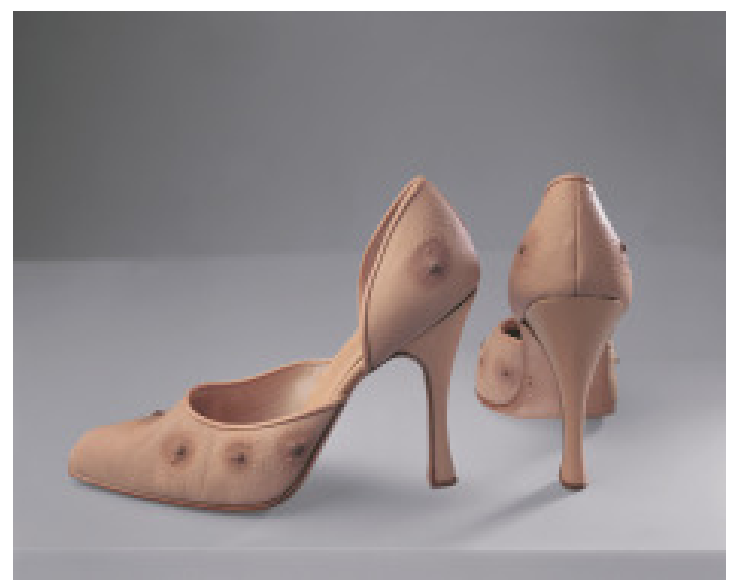

Figura 7. Peletería Humana, Nicola Costantino, 1996-2003.

\#PraTodoMundoVer Par de zapatos de taco alto de mujer en silicona, cubiertos con calcos de tetillas. 
Figura 8. Peletería Humana, Nicola Costantino, 1996-2003.

\#PraTodoMundoVer Par de zapatos de hombre en silicona cubiertos con calcos de anos.

La silicona imita la textura de la piel humana, e incluye como motivos decorativos anos, ombligos y tetillas masculinas. Este trabajo surgió a partir de la experimentación con la réplica en silicona de piel humana que la artista realizó en Houston School of Art en $1995^{2}$ - de hecho, en esa instancia Costantino visitaría Nueva York y Los Ángeles enfundada en un primer abrigo con ombligos y cabello natural, poniendo en escena una performatividad que resultaría central en la obra. La circulación de la obra fue significativa: Peletería humana sería presentada en la feria ARCO de Madrid por Ruth Benzacar en 1997, y al año siguiente Paula Herkenhoff llevaría la boutique de piel humana a la Bienal de Arte de San Pablo. En 1999 la obra volvería a Madrid, siendo seleccionada por Estrella de Diego para integrar la exposición Con los Cinco Sentidos en

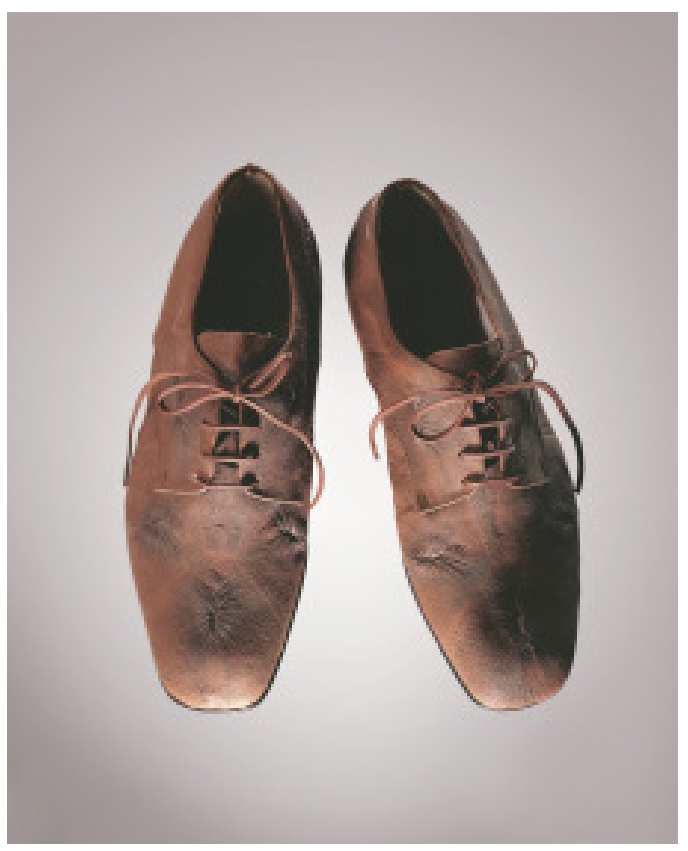
la Casa de América. En 2000, la peletería forma parte de la Bienal de Liverpool y de una muestra individual de Costantino en la galería de arte Deitch Projects, entre otros espacios -de hecho, cuando esta pieza se expuso allí, los transeúntes solían confundir la galería con una casa de moda de alta gama (Fig. 2). Así, la recepción inicial de la obra desestabilizaba los límites entre el arte y la moda, urgiendo a una reflexión sobre el consumo global de arte, la producción estética y la violencia al visibilizar los aspectos más profundamente abyectos de la moda femenina de lujo.

En esta línea, me interesa sugerir que mediante la recuperación de superficies corporales sensuales, táctiles y afectivamente cargadas que remiten a lo abyecto, Peletería humana negocia articulaciones hegemónicas de la sexualidad y el género e historias comunales de violencia, al tiempo que revisita imaginarios productivos y simbólicos nacionales para explorar, imaginar y delimitar nuevas corporalidades y subjetividades potenciales en torno tanto a lo femenino como a lo queer y lo viviente. En este sentido, Elizabeth Grosz se pregunta acerca de la relevancia de repensar ciertas nociones fundamentales del feminismo, como el concepto de 'libertad', atendiendo a la centralidad de lo material y lo natural en la constitución misma de la subjetividad libre o autónoma. Así, se pregunta: "ies el foco tradicional de la teoría feminista en la adquisición de la libertad de las mujeres de las restricciones patriarcales, racistas, colonialistas y heteronormativas el más adecuado? ¿O se trataría más bien de explorar lo que el sujeto femenino, o feminista, es y es capaz de hacer?" (Elizabeth GROSZ, 201 1 , p. 61). ${ }^{3}$ Lejos de despolitizar la lucha feminista, este reposicionamiento de la noción de 'libertad' se propondría "resituar al concepto... en un contexto diferente que pueda proveerle afiliaciones y asociaciones políticas alternativas, así como también una comprensión diferente de la subjetividad" (GROSZ, 201 1, p. 60). ${ }^{4}$ Mi interés en la obra de Costantino obedece, justamente, a cómo ésta investiga lo que un cuerpo es capaz de hacer, explorando sus potencialidades tanto a partir del tratamiento de su materialidad como de la técnica. En la obra de esta artista, el cuerpo sexuado se figura en flujo, en un devenir constante que (con)funde y cuestiona los límites subjetivos mediante el rescate de imaginarios, materialidades y procedimientos que refieren a lo trans, la androginia, lo femenino y lo viviente. En este sentido, se trataría menos de intentar percibir o comprender cada una de estas dimensiones experienciales desde sus propias lógicas que de indagar y expandir los modos en que toman forma en consonancia y en tensión unas con otras. En la obra de Costantino se plantea, así, un desplazamiento desde una política del reconocimiento (identitario) hacia una política de la inmersión, del estar con. Es a partir de ese estar con que su trabajo esboza una búsqueda centrada en devenires subjetivos orientados hacia lo no humano, una búsqueda que se enfoca en la investigación de nuevas formas de relación, intimidad, afectividad y conectividad entre corporalidades disímiles, y, desde esa plataforma, imagina otros modos de habitar el mundo.

\footnotetext{
${ }^{2}$ La obra Peletería Humana es "confección, cuerpo humano calcado con molde de alginato, más diseño en tela de silicona conforme a técnicas que...se usan en peletería" (Hans-Michael Herzog, 2013, p. 35).

${ }^{3}$ Todas las traducciones del inglés al español fueron realizadas por la autora. "Is feminist theory best served through its traditional focus on women's attainment of a freedom from patriarchal, racist, colonialist, heteronormative constraint? Or by exploring what the female - or feminist - subject is and is capable of making and doing?"

4 "reframing the concept... by providing it with a different context that may provide it with other, different political affiliations and associations and a different understanding of subjectivity."
} 


\section{La diferencia de género, la sexualidad y lo viviente}

Antes de enfocar mi análisis en Peletería humana, resulta importante señalar que el presente ensayo se enmarca en una lectura amplia de la obra de Costantino en tanto cuestionamiento sostenido de la categoría de lo humano como una vía posible desde donde pensar la diferencia de género y sexual. Entendemos el género y la sexualidad en términos foucaultianos, es decir, como construcciones biopolíticas sujetas a constante regulación y disciplinamiento que, asimismo, expanden su alcance y toman forma con relación a lo viviente y a lo inerte. Como afirma el crítico norteamericano Mel Chen, "Ios modos en que los objetos inanimados y los animales no humanos participan en los regímenes de la vida (hacer vivir) y la muerte coercitiva (dar muerte) son integrales al esfuerzo de comprender cómo funciona el biopoder y cuáles son sus materiales" (Mel CHEN, 2012, p. 6). En esta línea, la serie de obras de Costantino que indagan los límites entre lo animal y lo humano bajo el lente de la diferencia de género y sexual es extensa. De madre costurera y padre cirujano, Costantino parece consumar ambos oficios en la realización de Bolas (1998-2004) (que incluyen 'ternerobolas', 'chanchobolas', 'pollobolas' e incluso 'ñandubolas') y Cajas (20002005), que junto con Cochon sur canapé (1992), Animal Motion Planet (2004), Frisos (2000-2008), Carneada (2000-2002) y su serie fotográfica Alteridad (2012) profundizarían algunas de estas líneas de trabajo. ${ }^{6}$ Resulta relevante que los animales que emergen, insistentes, en la obra de Costantino son a menudo aquellos mismos que encontramos al adentrarnos en el territorio argentino y, en particular, en la Pampa en tanto espacio privilegiado de producción agrícola y ganadera. Su obra evoca, así, violencias fundacionales asociadas al espacio del campo y la producción violencias marcadas en términos no sólo de especie sino también de raza, clase, género y sexualidad - que dan forma e impulso al proyecto país.

El límite difuso entre la existencia humana y lo que Giorgio Agamben denomina 'nuda vida' emerge a la vez como interrogante en su controversial Savon de corps (2004, Fig. 9), una videoinstalación que consistió en la elaboración de cien jabones con un porcentaje de grasa corporal de la artista obtenida de una liposucción a la que se sometió para este proyecto.

Cual lanzamiento de un artículo cosmético de lujo, la artista, sugerente, invita al público a

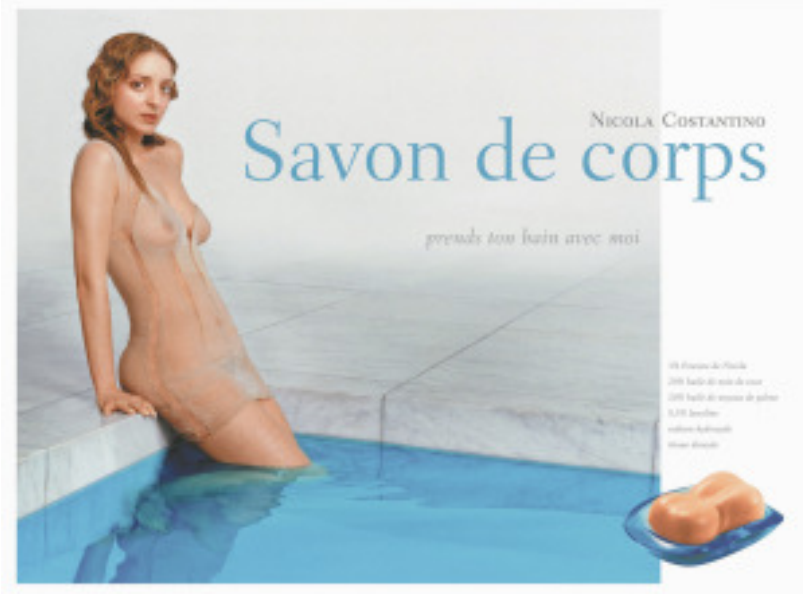

Figura 9. Savon de corps, Nicola Costantino, 1996-2003.

\#PraTodoMundoVer Figura la artista vestida con una camisola transparente dentro de una piscina, apoyada contra el borde exterior de la misma y mirando a cámara. La imagen es acompañada por un texto que reproduce el nombre de la obra, de la artista y, en francés, se invita al espectador a tomar un baño con ella ("prend ton bain avec moi"). En el extremo derecho inferior de la imagen se muestra un jabón color piel sobre una jabonera celeste.

"tomar un baño" con ella. Para articular el concepto de 'nuda vida', Agamben se sirve de la distinción realizada por Aristóteles entre bíos y zoé: mientras bíos denomina a aquella vida que posibilita lo social y, consecuentemente, lo político (en otras palabras, un tipo de existencia o vida cualificada sólo propia del hombre), zoé sería la pura vida biológica, la mera condición de existir común al ser humano y lo viviente. En este sentido, zoé es aquello que une y conecta al ser humano

\footnotetext{
5 "how inanimate objects and nonhuman animals participate in the regimes of life and coerced death (killing) are integral to the effort to understand how biopower works and what its materials are."

${ }^{6}$ La indagación de Costantino en torno a lo animal forma parte de una serie de producciones visuales latinoamericanas contemporáneas que han abordado esta temática. Mientras que en Chile, por ejemplo, Demian Schopf trabaja con animales embalsamados y en descomposición en La revolución silenciosa (2001-2002) y Gabriela Rivera explora los vínculos estéticos y políticos entre el cadáver animal y la corporalidad femenina, en Argentina surgen una serie de trabajos como el de Luis Fernando Benedit, quien explora la figura del gaucho y el animal en el contexto de la pampa; Marcelo Pombo, cuya articulación de lo animal, lo vegetal y los lúdicos cruces entre lo animal y lo humano que derivan en la exploración de figuras humanoides; Fernanda Laguna, que en la línea de Pombo trabaja las formas orgánicas y lo animal desde el registro de lo ingenuo, y Leo Chiachio y Daniel Giannone, donde lo animal emerge en el ámbito de lo doméstico para sugerir nuevos abordajes queer a la noción de familia. A su vez, la propuesta estética de Costantino en torno a la animalidad encuentra correlatos en trabajos como el de la mejicana Teresa Margolles y el colectivo SEMEFO (Servicio Médico Forense) en los años 90, que visibilizan la (bio)política de los restos.
} 
con lo animal y lo vegetal, es decir, la vida entendida como materia. Una vida sin forma humana, o 'nuda vida', sería entonces vida sin ningún tipo de estatuto político (siendo la política una actividad humana) y, consecuentemente, sin estatuto jurídico (Giorgio AGAMBEN, 2006, p. 17-8). En este contexto, lo político se concibe como:

[...] el lugar en el que el mero vivir debe convertirse en bien vivir, como el lugar en el que el hombre, definido como "animal viviente y, además, capaz de existencia política", debe suprimir aquello que lo caracteriza como mero viviente para realizar aquello que lo distingue como hombre. La relación de inclusión por exclusión, o excepción, indica entonces que hay algo que se presupone (en este sentido se incluye) como aquello que hay que suprimir, superar (en este sentido se excluye), para pertenecer a la vida política...En este sentido Agamben señala "Ia 'politización' de la nuda vida es la tarea metafísica por excelencia en la cual se decide acerca de la humanidad del ser vivo hombre". Y con ello la pertenencia y la no pertenencia, el afuera y el adentro de la comunidad humana. (Laura QUINTANA PORRAS, 2006)

La pregunta en torno a la 'nuda vida', y su insistente continuidad con modos de vida cualificados como humanos, resulta central en Savon de corps. El foco en el jabón a base de grasa humana desató una polémica a nivel nacional debido a la potencial conexión de la obra con la historia del Holocausto, dando paso a una recepción marcada fuertemente por la dimensión emocional y caracterizada por el rechazo, el asco y la abyección. Es allí también a partir de una pieza con gestos performáticos que remiten a la materialidad y la vida del cuerpo femenino de la artista que se articula la pregunta, central en la obra de Costantino, en torno al estatuto de lo humano. Siguiendo esta línea de pensamiento, la emergencia del cabello humano en Peletería humana - quizás el material más abyecto y repulsivo desplegado en la obra al presentarse, en tanto resto biológico, con absoluta literalidad y sin la mediación técnica del calco - anticiparía la pregunta que emergerá más tarde en Savon de corps acerca de los usos de la materia orgánica humana en el contexto del nazismo, los imaginarios y mitos en torno a la misma, y su lugar en los procesos posteriores de memorialización. ${ }^{7}$ El estado de excepción en tanto problema político y estético emerge, así, de manera sostenida a través del trabajo de la artista, complejizado por un impulso que parece simultáneamente tender a la espectacularización a partir no solo de los recursos técnicos desplegados, sino también del lenguaje comercial de la publicidad y la moda.

Retomando la importancia de lo viviente para problematizar el estatuto de lo humano y, en particular, las limitaciones de esta última categoría a la hora de definir y legitimar subjetividades marcadas en términos de género y sexualidad, es importante señalar la centralidad de la muerte animal en la constitución misma de los imaginarios nacionales argentinos. Gabriel Giorgi rescata la tradición de la representación del matadero en la literatura local, cuyo gesto fundante lo realiza Esteban Echeverría en el siglo XIX (1838-40) y es continuado por Osvaldo Lamborghini, Rodolfo Walsh y Pino Solanas, entre otros, en tanto espacios de saber biopolítico que desterritorializan y ponen en crisis la ontología de lo humano y lo animal (Gabriel GIORGI, 2014). Según Giorgi, la cultura inscribió los mataderos y los volvió un lugar clave para pensar la relación entre animalidad y política en consonancia con dos modos de política: por un lado, "Ia política 'arcaizante', barbarizante y premoderna de la violencia soberana" que se exhibe por excelencia en el matadero, y por otro "Ias políticas 'modernizadoras' del capital en tanto "máquina o dispositivo biopolítico que enlaza los cuerpos - de los animales y de los trabajadores - en torno a la producción de la carne como mercancía" y la consecuente instrumentalización de la capitalización de la vida y de la muerte (GIORGI, 2014, p. 134). Ambos modos de (bio)política se encuentran latentes en estas obras de Costantino. ${ }^{8}$ A la vez, como señala David Viñas en su clásica observación crítica en referencia a El matadero de Esteban Echeverría, el gesto fundante de la literatura argentina es nada más y nada menos que una violación. De esta forma, las lógicas de la capitalización de la vida y de la muerte en torno a lo animal y a lo humano puestas en marcha por el dispositivo del matadero en pos de la producción se entrelazan con las de la violencia sexual. En esta línea, en las obras de Costantino los cuerpos animales maniatados reflejan y visibilizan violencias de género, retomando la problemática de la reproducción mediante la constante tematización de animales nonatos y su estrecho vínculo con los imaginarios productivos nacionales. ${ }^{9}$

\footnotetext{
7 El Museo y Memorial Auschwitz-Birkenau en Polonia preserva en exhibición cabello humano perteneciente a las víctimas del Holocausto. El cabello humano era obtenido de los cuerpos de los judíos asesinados luego de su paso por las cámaras de gas. http://auschwitz.org/en/gallery/exhibits/evidence-of-crimes, 1.html

${ }^{8}$ Las referencias directas e implícitas tanto al espacio, las prácticas y estética del matadero como a los procesos de producción y comercialización que de éste derivan resultan una constante a través del trabajo de Costantino. Obras fundamentales en su producción como Cochon sur canapé (1992), Cajas (2000-2005) y Bolas (1998-2004), Carneada (2000-2002), Frisos (2000-2008) y Animal Motion Planet (2004) se centran justamente en esta problemática.

9 Ver, por ejemplo, sus series Bolas (1998-2004), Cajas (2000-2005) y Frisos $(2000-2008)$ y su obra Limbo de nonatos (Nonatividad) (1999).
} 


\section{'Conceptos matéricos' y comunidades expandidas: Tecnologías, afectos e imaginarios de lo trans}

Atendiendo a las tecnologías que posibilitan la muerte animal a gran escala, una parte significativa de la serie de animales de Costantino recrea complejas maquinarias que re-imaginan las potencialidades de la técnica en torno a la materia, jugando, como señala la artista, con las figuras del autómata y lo maquínico, y a partir de ese imaginario, con la ilusión de "devolverles la vida a los muertos" (COSTANTINO, 2017). La máquina como objeto de indagación artística, una constante en la obra de Costantino, evoca de manera insistente las tecnologías del matadero y su sistematización de la muerte, al tiempo que investiga el universo de la técnica, aspecto también central en su trabajo. En esta línea, deteniéndome en las superficies y texturas corporales desplegadas, propongo aquí una lectura que, más que descansar en la intencionalidad de la artista, explora las derivas y sentidos potenciales asociados a la técnica, los lenguajes, los afectos y la materialidad que la propia obra moviliza. La propia Costantino señala la primacía de lo matérico y lo técnico por sobre cualquier idea fundante en su proceso de producción artística:

Yo creo que las ideas para mis obras surgen a partir de las técnicas y los materiales con los que trabajo. Mis conceptos, ya de por sí 'matéricos', vienen con el medio para realizarlos incorporado. Incluso puedo pensar mis obras como el resultado de una ecuación cuyos factores son técnicas que he ido aprendiendo a dominar. (HERZOG, 2013, p. 35)

De hecho, el minucioso trabajo técnico basado en la química, la mecánica, la costura, el calco y la matricería, así como la condición de artificialidad y artefacto que caracterizan la obra de Costantino, pueden relacionarse con la necesidad de generar un grado de distanciamiento de la abyección que las piezas despliegan. Ese distanciamiento sería necesario, según la artista, para dar lugar a un modo de recepción de la obra capaz de propiciar una conexión con la materialidad y las problemáticas trabajadas que no se agote meramente en el rechazo visceral. ${ }^{10}$ Se trataría, en otras palabras, de un intento por modular la intensidad de la reacción afectiva del público para encontrar el punto exacto en que su obra pueda contemplarse y experimentarse sin perder del todo la sensación de asco y abyección (y el simultáneo goce estético) en el centro mismo de la propuesta.

En esta línea, en Peletería humana la ilusión de "devolverles la vida a los muertos" a la que refiere Costantino se ve reforzada por la referencia a la creación de un ser a partir de partes de cuerpos anónimos representada culturalmente en el monstruo de Frankenstein. La artista recoge así, en el código del lenguaje fotográfico con el que el calco dialoga, la "presencia de lo que ya no está" (COSTANTINO, 2017), performativizando, podemos pensar, una iteración posible de un duelo colectivo - un duelo desde un cuerpo que es una multitud, un ensamblaje. El calco detallado de corporalidades anónimas, con sus poros y sus marcas, de la piel como superficie de inscripción de una historia personal y compartida, opera aquí como un lenguaje a partir del cual sostener la presencia de los que ya no están a través de la materialidad de sus huellas físicas, su registro indexical. Como parte de un ejercicio de recuperación, un procedimiento indexical característico de la fotografía, otro de los registros a los que constantemente retorna la obra de Costantino, se desplaza aquí hacia la escultura. ${ }^{11}$

Así, es posible detectar una insistencia en corporalidades (humanas y animales) fuertemente intervenidas en la obra de Costantino, donde tanto el ser vivo como el cadáver emergen como materia plástica siempre plausible de ser moldeada, violentada y reconfigurada. Como señalé anteriormente, en Peletería humana Costantino crea prendas que presentan tetillas masculinas, ombligos y anos sobre su superficie. Si por un lado esas prendas, diseñadas para ser usadas por mujeres y hombres, visibilizan y problematizan los vínculos entre lo animal y lo humano en el contexto de la producción agropecuaria de la Pampa, refieren a la vez insistentemente al género y la sexualidad. Evocando lúdicamente el universo de la cirugía estética -un universo que entra en tensión, a su vez, a partir del acercamiento personal de la propia artista a ese tipo de

\footnotetext{
${ }^{10}$ Ese impulso deriva en el uso de la técnica del calco en la obra de Costantino. Según la artista, "...para cuando llegué al Taller de Barracas yo ya había trabajado con animales momificados en 1992, pero venía con el problema de la conservación de la obra: mis animales se degradaban y desagradaban. En ese momento me empecé a dar cuenta de que ese tipo de técnica impresionaba mucho, producía un rechazo y un asco que de alguna manera impedía que el público se conectara con el sentido de la obra. Advertir ese asco del público fue un aprendizaje para mi, porque yo nunca lo sentí de esa manera. Lo que quería era que la obra se entendiera, así que busqué la forma de presentar al animal de manera realista, pero prescindiendo del cuerpo verdadero, que es lo que puede causar asco." (HERZOG, 2013, p. 25).

${ }^{11}$ Costantino comienza a utilizar la técnica del calco de manera sostenida a partir del desarrollo de la misma en su obra Chancho con motor en 1994. En sus palabras, "aunque el público sabe que está ante una reproducción en resina, el calco da la sensación de que se está frente al cuerpo real, por la perfección de la textura de la piel y la reproducción de los poros, los brillos, las rugosidades, los pliegues. Así como al mirar una fotografía de un lugar uno tiene la fantasía de 'conocerlo', de haber tenido un contacto directo con lo que aparece en la imagen, los calcos nos hacen experimentar la presencia del animal y al mismo tiempo resguardan al público de lo que produce asco: la carne, las plumas, los pelos..." (HERZOG, 2013, p. 25).
} 
procedimientos-, ${ }^{12}$ Peletería humana presenta una corporalidad múltiple y en perpetua construcción a partir de los procedimientos del calco, el recorte, la costura y el ensamblaje. Una corporalidad que lleva las marcas de una puesta en escena, una performatividad que se reitera como tropo en gran parte de su obra y que resulta central a la hora de dar cuenta de su intervención con relación a la problemática de género. ${ }^{13}$ En su fundacional El género en disputa. El feminismo y la subversión de la identidad, Judith Butler argumenta que el género, lejos de una categoría esencial o natural, es performativo. En otras palabras, el género se produciría como efecto de la repetición sostenida y ritualizada de convenciones sociales que obedecen a la heterosexualidad hegemónica. Así la performance de género, en tanto actuación reiterada y obligatoria sobre la base de normas que exceden al individuo, produciría el efecto de una esencia natural o disposición original y verdadera. En palabras de la autora, "la univocidad de género, la coherencia interna del género y el marco binario para sexo y género son ficciones reguladoras que refuerzan y naturalizan los regímenes de poder convergentes de la opresión masculina y heterosexista" (Judith BUTLER, 2007, p. 99). La consecuente crítica de Butler a las categorías de identidad, consideradas entidades fijas limitantes de las posibilidades de agencia que surgen al entender el género como un efecto producido culturalmente, deriva en la propuesta de una multiplicación radical del género capaz de problematizar sus normas. Esa disrupción de las nociones de género y sexo naturalizadas ocurriría a partir de "la proliferación paródica y la interacción subversiva de significados con género" (BUTLER, 2007, p. 99) que moviliza, multiplica y confunde las categorías constitutivas del género socialmente codificado. La obra de Costantino parece hacer eco de estas reflexiones al re-imaginar, a través de un gesto performático, distribuciones posibles de los aspectos físicos y simbólicos que constituyen el sexo y el género, multiplicando sus sentidos mediante una ilegibilidad radical que atraviesa, incluso, las fronteras de lo humano. De este modo la noción de performatividad de género, reforzada mediante el gesto físico del uso de las prendas por parte de las modelos y de la propia artista, se redefine y se vuelve múltiple e inclasificable.

En Peletería humana, esa ilegibilidad con relación a lo sexo-genérico evoca, confunde y multiplica una variedad de corporalidades, imaginarios, afectos y deseos asociados, entre otros, al cuerpo femenino, lo queer, la androginia y lo trans. Esta proliferación desordenada interpela las normas de constitución de identidad de género al tiempo que sugiere nuevas potencialidades de lo corpóreo y otras concepciones de lo colectivo. En este sentido, la obra puede leerse como un acercamiento literal y matérico a las transformaciones potenciales del cuerpo sexuado. Si el cuerpo andrógino emerge en la obra a partir del despliegue indexical de partes corporales sexuales múltiples y no diferenciadas, ${ }^{14}$ los imaginarios vinculados a lo trans se encarnan, de manera crucial, a través del gesto físico y simbólico de intervención y transformación condensado en la acción de ensamblar, coser, unir y poner en relación (y en tensión) esas corporalidades. La piel en tanto barrera porosa se explora como superficie de contacto y conexión, potencial barrera comunicante entre cuerpos y espacio fronterizo de re-configuración y re-invención subjetiva y comunitaria.

Es importante consignar que tanto la androginia como lo trans constituyen una preocupación a través de la obra de Costantino. ${ }^{15}$ Atendiendo a la indagación sostenida de la problemática de

\footnotetext{
${ }^{12}$ Lejos de una posición ecológica o activista, Costantino exhibe las contradicciones que dan forma a su arte y a nuestros intercambios con lo animal, definidos por la intimidad promiscua de la muerte, el deseo y el placer. La artista se posiciona con relación a su obra en estos términos: "yo no emito juicios sobre las contradicciones que muestro porque generalmente yo misma protagonizo el comportamiento que podría resultar criticable: yo soy la que come carne, soy la que se somete a una cirugía estética..." (citada en HERZOG, 2013, p. 26).

${ }^{13}$ La dimensión performática de la obra de Costantino se solidifica en particular en sus series fotográficas posteriores a partir del procedimiento de la cita de obras canónicas de la historia del arte. Consultar las obras Nicola en el lago (2007), Madonna (2007) y Nicola alada, inspirada en Bacon (2010) para una aproximación al tratamiento del vínculo entre la corporalidad femenina, la performance y lo animal en sus series fotográficas.

${ }^{14}$ Como explica Costantino, "los calcos fueron realizados en distintos momentos a diferentes personas, las tetillas son todas masculinas, los ombligos y anos no discriminan sexo así que nunca digo el género del original porque no importa" (COSTANTINO, 2019).

${ }^{15}$ En su reciente obra El verdadero jardín nunca es verde (2016, Galería Barro y 2017, Centro Cultural Kirchner), la artista sugiere corporalidades andróginas en el contexto de escenas que reponen socialidades tradicionalmente femeninas como la maternidad, la cocina y la transmisión oral. Una serie de cuerpos vestidos de manera uniforme con mallas de lycra y cofias lúdicamente encarnan una obra de Costantino de 1997, Trilogía de bocas: de sus bocas humanas emerge un hocico de cerdo, de cuyo cogote cuelga una cabeza y cuello de pollo. Así, la obra indaga tanto la diferencia de género como la potencialidad de la trans-especie en una atmósfera que, reforzada por una imagen de Jerusalén en llamas, la artista define como posapocalíptica (COSTANTINO, 2019). En Pardés (2018), una versión posterior de esta obra presentada en la Usina del Arte, a los personajes andróginos que preparan un banquete se integra como protagonista Leandra, una mujer trans vestida de orquídea con un traje de organza transparente. A partir del trabajo con la variedad de orquídea denominada 'ghost', que se asemeja en su forma a un insecto o un animal en estrecha relación con el cuerpo de Leandra, la obra imagina otras distribuciones posibles de la multiplicidad identitaria y sus atributos más allá de la noción de género y de la subjetividad humana. En palabras de Costantino, "se trata de una orquídea que está en vías de extinción y actualmente es salvaje, se encuentra solo en unos pantanos en la zona de Florida y es tan impresionante porque tiene una cosa medio de bicho, la ves y decís 'esto no es una flor'. Es una flor donde se mezcla el límite de lo vegetal y lo animal, y además las orquídeas tienen sus colores y sus formas para seducir insectos... Entonces la orquídea para mí es este símbolo de algo que traspasa las especies, y la Leandra acá está transformada en orquídea" (COSTANTINO, 2019).
} 
género y sexualidad en su trabajo, me interesa seguir aquí la pista de los modos en que su obra trabaja lo trans en tanto instancia paradigmática de la identidad en flujo y de una corporalidad en constante devenir. Una corporalidad que transiciona, se re-inventa y recrea sus límites hasta derivar en la trans-especie, en la medida en que el juego en torno al despliegue de la estética y la técnica de la peletería animal a partir del calco de piel humana horada los límites entre la vida imbuida de derechos y la nuda vida, el cuerpo animal y el humano en tanto materia prima para la producción. Y es justamente a través del despliegue de la técnica que Peletería humana propone una reflexión sobre tecnologías de transformación y re-invención corporal que, como el corte y la reparación, resultan centrales al proceso de reconfiguración física de algunos individuos que transicionan. En otras palabras, las técnicas asociadas a la manipulación e intervención de los cuerpos que pueblan la obra de Costantino (técnicas que la artista asocia con su entorno familiar y, en particular, con la influencia de su madre costurera y su padre cirujano), así como su trabajo con la materia en tanto entidad plástica, la vuelven un espacio fértil de reflexión sobre procesos de reconfiguración corporal.

En esta línea, a partir de un detallado análisis de la canción The Cripple and the Starfish ("El discapacitado o inválido y la estrella de mar", 2000), de Antony and the Johnsons, la crítica trans Eva Hayward propone que el corte y la cicatriz implicados en la reconfiguración y transformación corporal de lo viviente y, en particular, de la estrella de mar, pueden ayudarnos a pensar los modos de corporalización característicos de los individuos trans. La estrella de mar en tanto objeto material y discursivo, propone Hayward, es una figura interesante para pensar procesos de corporalización. Este equinodermo es capaz de reproducirse sexual y asexualmente - en el primer caso, la fertilización tiene lugar externamente a medida que los especímenes masculinos y femeninos liberan sus gametos al medio ambiente. Algunas especies de estrella de mar se reproducen de manera asexuada mediante la fisión, que a menudo implica que parte del cuerpo de un espécimen se desprende y eventualmente se desarrolla en un nuevo individuo. Si bien la mayoría de las especies necesitan mantener la parte central de su cuerpo intacta para poder regenerarse, unas pocas son capaces de producir una estrella de mar completa a partir de un solo brazo o rayo. Atendiendo a la canción de Antony and the Johnsons y siguiendo el modelo de la estrella de mar, Hayward entiende el corte como un acto generativo de reparación física que materializa la posibilidad de reconfiguración, regeneración y reinvención corporal trans a partir de la herida. El corte activaría, así, la corporalización trans

[...] al remitir no necesariamente a la castración sino más bien al intento de reconfigurar el 'yo' mediante esa intervención corporal... El corte es posibilidad. Para algunas mujeres transexuales, el corte es menos una apertura del cuerpo que un esfuerzo generativo de retraer el cuerpo hacia y a través de si mismo para sentir la reparación y el crecimiento de nuevos márgenes. El corte no es solo una acción: el corte es parte del proceso constante de materialización mediante el cual un individuo transexual tentativamente deviene. (Eva HAYWARD, 2008, p. 71-2) ${ }^{16}$

En esta misma línea y refiriendo a la capacidad transformadora de lo artístico, una capacidad que evade las clasificaciones estancas y que remite a procesos en constante flujo y devenir que, según Antony, "comienzan en un lugar y terminan en otro", Hayward propone que el prefijo "trans", cargado con el sentido de atravesar, trascender o ir más allá de, sugiere lo inclasificable: "ser trans es encontrarse en proceso de trascender o superar imposiciones específicas, ya sean de carácter empírico, retórico o estético" (HAYWARD, 2008, p. 68). ${ }^{17}$

La obra de Costantino, constituida a partir de una técnica de (re)corte, yuxtaposición y combinación, resuena junto a estas ideas de Hayward, quien hace referencia al 'collage' en tanto manifestación estética de los modos en que "Ias transformaciones de todo tipo, de manera similar a los individuos transgénero, son producidas a través de fuerzas afectivas. Piezas y retazos (nuevamente, de algo roto) son recombinados en nuevas formas integrales de sentido que no constituyen, sin embargo, totalidades" (HAYWARD, 2008, p. 68). ${ }^{18}$ Peletería humana sugiere, en esta línea, una corporalidad mutable y fluida, en constante devenir. Asimismo, el eco de la transsexualidad deriva aquí en la trans-especie: la obra evoca los procesos productivos, simbólicos y estéticos asociados a la corporalidad animal para re-desplegarlos en relación a la materialidad humana. En esta línea, Eva Hayward lee el álbum I am a Bird now (Soy un pájaro ahora) de Antony and the Johnsons, incluido como instalación en la Bienal Whitney de 2004, como una propuesta que aboga por la "transformación como tropo para reconfigurar la relacionalidad entre lo femenino y lo masculino, lo humano y lo animal" (HAYWARD, 2008, p. 68): ${ }^{19}$

\footnotetext{
16 "to cut is not necessarily about castration, but an attempt to recast the self through the cut body... The cut is possibility. For some transsexual women, the cut is not so much an opening of the body, but a generative effort to pull the body back through itself in order to feel mending, to feel the growth of new margins. The cut is not just an action; the cut is part of the ongoing materialization by which a transsexual tentatively and mutably becomes."

17 "to be trans- is to be transcending or surpassing particular impositions, whether empirical, rhetorical, or aesthetic." 18 "transformations - not unlike transgenders - are produced through emotive forces. Shards and pieces (again, of something broken) are reworked into meaningful integrities, but now wholes."

19 "transformation as a trope for reworking the relationality of male and female, of human and animal."
} 
El prefijo 'trans-', como lo expresa Antony, está destinado a perturbar las prácticas de purificación, lo bien definido se confunde en múltiples niveles materiales y semióticos. Las experiencias psíquicas y corporales se entremezclan. Por ejemplo, el género y la corporización del género son contingencias que pueden sostenerse por un momento para luego derivar en otro conjunto de relaciones. Las especies existen a partir de sus diferencias taxonómicas (Homo sapiens sapiens no es lo mismo que Octopus vulgaris), pero a la vez las especies siempre ya se constituyen mutuamente a través de los espacios y lugares que co-habitan, incluyendo el lenguaje y otros registros semióticos (Haraway, 2003)... La materia no es inmutable, sugiere Antony, sino discursiva, permitiendo que los sexos y las especies practiquen la trans-materialización. La carne y el significado para los humanos y las estrellas de mar no se asientan en una falta estructural, ni en una división primordial, sino que se entrelazan sensualmente. (HAYWARD, 2008, p. 69) ${ }^{20}$

En este sentido, y siguiendo a Hayward en su definición de la sexualidad trans en relación a la materialidad de otras especies animales y, en particular, a la capacidad de regeneración de la estrella de mar, la obra de Costantino resuena con la afirmación de Donna Haraway acerca de los modos en que "Ias especies de todo tipo se constituyen mediante encuentros" (HAYWARD, 2008, p. 70). ${ }^{2{ }^{2}}$ En otras palabras, "no somos humanos solos sino con muchos otros" (HAYWARD, 2008, p. 69). ${ }^{22}$ Reconstituyendo relacionalidades múltiples y complejas entre lo femenino, lo masculino, lo humano y lo animal, me interesa sugerir entonces que Peletería Humana propone un territorio común desde el que se evocan diversas corporalidades a partir del recorte, el ensamblaje y la regeneración en un contexto contemporáneo en que las tecnologías biomédicas, la cirugía plástica y otros modos de reconfiguración corporal desdibujan de manera insistente las fronteras de lo humano. Ese territorio de emergencia de la diversidad y la continuidad material y simbólica de lo viviente se articula mediante una apuesta formal que delinea, a su vez, los trazos tentativos de una estética.

A modo de conclusión, es posible leer en la yuxtaposición de partes corporales indiferenciadas que se pliegan unas sobre otras en Peletería humana la emergencia de una corporalidad múltiple y anti-normativa que remite a lo común. No se trataría ya de un cuerpo, o de sus rastros, sino más bien de un ensamblaje que denota una preocupación constante por modos otros de lo colectivo. Retomando las palabras de la artista en torno a Peletería humana y su interés en explorar la violencia de la dimensión del consumo, es posible leer en la pieza una evocación del mundo y los cuerpos marginados de la producción agropecuaria y sus imaginarios nacionales. En esta línea, los modos otros de relacionalidad (y continuidad) entre lo animal y los cuerpos disidentes que la obra explora a partir de los tropos de lo trans se asientan simbólicamente también, mediante el recurso técnico del calco, en la reconstrucción metonímica de cuerpos ausentes cuerpos maniatados, marginados y monstruosos que acarrean sobre su superficie las marcas de violencias fundacionales. Mediante un gesto performático que alude a la magia en su intención de transmutar y 'devolverle la vida a los muertos' $y$, en términos de Hayward, a un modo posible de transformación estética, Peletería humana memorializa cuerpos ausentes al tiempo que materializa corporalidades híbridas y ensambladas para, trazando alianzas trans-subjetivas y trans-especie, re-imaginar las potencialidades de la corporalización sexo-genérica a la hora de desajustar modelos normativos y opresivos de identidad, pertenencia y nación.

\section{Referencias}

AGAMBEN, Giorgio. Homo sacer: El poder soberano y la nuda vida. Valencia: Pre-textos, 2006.

ANTONY AND THE JOHNSONS. 2000. "The Cripple and the Starfish". Rebis Music (SC104). Originalmente lanzada el 1 de Mayo, 2000.

BRAIDOTTI, Rosi. Lo posthumano. Barcelona: Editorial Gedisa, 2015.

BUTLER, Judith. El género en disputa: El feminismo y la subversión de la identidad. Barcelona: Ediciones Paidos, 2007.

CHEN, Mel. Animacies: Biopolitics, Racial Mattering and Queer Affect. Durham: Duke University Press, 2012.

\footnotetext{
20 "Trans-," as articulated by Antony, is meant to disturb purification practices; the well defined is confounded at multiple material and semiotic levels. Psychical and corporeal experiences are blended. For example, gender and the embodiment of gender are contingencies that may hold for a moment then fall away into another set of relationships. Species exist in taxonomic differences (Homo sapiens sapiens is not the same as Octopus vulgaris), but species are also always already constitutive of each other through the spaces and places we cohabit - this of course includes language and other semiotic registers (Haraway 2003)... Matter is not immutable, suggests Antony, it is discursive, allowing sexes and species to practice trans-materialization. The meat and meaning for humans and starfish have no structuring lack, no primordial division, but are sensuously intertwined."

21 "species of all sorts are constituted through encounters."

22 "we are not human alone - we are human with many."
} 
COSTANTINO, Nicola. Cochon sur canapé, 1992.

COSTANTINO, Nicola. Chancho con motor, 1994.

COSTANTINO, Nicola. Peletería humana, 1996-2003.

COSTANTINO, Nicola. Bolas, 1998-2004.

COSTANTINO, Nicola. Trilogía de bocas, 1997.

COSTANTINO, Nicola. Limbo de nonatos (Nonatividad), 1999.

COSTANTINO, Nicola. Ternerobolas, 2000.

COSTANTINO, Nicola. Carneada, 2000-2002.

COSTANTINO, Nicola. Cajas, 2000-2005.

COSTANTINO, Nicola. Frisos, 2000-2008.

COSTANTINO, Nicola. Animal Motion Planet, 2004.

COSTANTINO, Nicola. Nicola en el lago, 2007.

COSTANTINO, Nicola. Madonna, 2007.

COSTANTINO, Nicola. Nicola alada, inspirada en Bacon, 2010.

COSTANTINO, Nicola. Alteridad, 2012.

COSTANTINO, Nicola. El verdadero jardín nunca es verde, 2016-2017.

COSTANTINO, Nicola. Pardés, 2018.

COSTANTINO, Nicola. Entrevista por la autora. 2017.

COSTANTINO, Nicola. Entrevista por la autora. 2019.

GIORGI, Gabriel. Formas Comunes: Animalidad, Cultura, Biopolítica. Buenos Aires: Eterna Cadencia, 2014.

GROSZ, Elizabeth. Becoming undone. Darwinian Reflections on Life, Politics and Art. Durham: Duke University Press, 2011.

HARAWAY, Donna. The Companion Species Manifesto: Dogs, People, and Significant Otherness. Chicago: Prickly Paradigm Press, 2003.

HAYWARD, Eva. "More Lessons from a Starfish: Prefixial Flesh and Transspeciated Selves". Women's Studies Quarterly, Nueva York, v. 36, n. 3/4, p. 64-85, otoño-invierno 2008.

HERZOG, Hans-Michael. "Entrevista”. In: Nicola Costantino. Alemania: Hatje Cantz Verlag, 2013. p. 20-36.

QUINTANA PORRAS, Laura. "De la nuda vida a la 'forma-de-vida'. Pensar la política con Agamben desde y más allá del paradigma del bio-poder". Argumentos, Ciudad de México, v. 19, n. 52, p. 4360, septiembre/diciembre, 2006. Disponible en: http://www.scielo.org.mx/scielo.php?script=sci_arttex t\&pid $=$ S0187-57952006000300003. Acceso 20/08/19.

SCHOPF, Demian. La revolución silenciosa, 2001-2002.

Cynthia Francica (cynthiafrancica@gmail.com) es Doctora en Literatura Comparada por la Universidad de Texas en Austin, y posee un Máster en Literatura Comparada otorgado por la misma universidad. Actualmente es Directora del Magíster en Literatura Comparada e investigadora del Centro de Estudios Americanos (CEA) de la Universidad Adolfo lbáñez en Santiago, Chile. Ha publicado sobre arte, performance y literatura latinoamericana en diversos medios, incluyendo Journal of Latin American Cultural Studies (JLACS), Alter/nativas: Latin American Cultural Studies Journal, Estudios Avanzados (USACH) y Estudios Filológicos. 
COMO CITAR ESTE ARTÍCULO, DE ACUERDO CON LAS NORMAS DE LA REVISTA:

FRANCICA, Cynthia. "Animalidad, comunidad e imaginarios de lo trans en la obra visual de Nicola Costantino". Revista Estudos Feministas, Florianópolis, v. 28, n. 2, e72439, 2020.

\section{CONTRIBUCIÓN DE AUTORÍA}

No se aplica

\section{FINANCIACIÓN}

El presente artículo forma parte del proyecto ANID/CONICYT FONDECYT No 11180229 , "Más allá de lo humano: cuerpo femenino y afecto en la literatura y las artes visuales contemporáneas de Chile y Argentina", a cargo de Cynthia Francica.

CONSENTIMIENTO DE USO DE IMAGEN

Autorizado por el Estudio Nicola Costantino.

APROBACIÓN DE COMITÉ DE ÉTICA EN INVESTIGACIÓN

No se aplica.

CONFLICTO DE INTERESES

No se aplica.

\section{LICENCIA DE USO}

Este artículo está licenciado bajo la Licencia Creative Commons CC-BY Internacional. Con esta licencia se puede compartir, adaptar, crear material para cualquier objetivo, siempre que se le atribuya la autoría.

\section{HISTORIAL}

Recibido el 25/03/2020

Aprobado el 06/05/2020 